Newfoundland and Labrador Studies

\title{
Étude du risque dans la vie maritime autour de Saint-Pierre-et-Miquelon 1817-1948
}

\section{Nicolas Landry}

Volume 34, Number 2, 2019

URI: https://id.erudit.org/iderudit/1072652ar

DOI: https://doi.org/10.7202/1072652ar

See table of contents

Publisher(s)

Faculty of Arts, Memorial University

ISSN

1719-1726 (print)

1715-1430 (digital)

Explore this journal

Cite this article

Landry, N. (2019). Étude du risque dans la vie maritime autour de

Saint-Pierre-et-Miquelon 1817-1948. Newfoundland and Labrador Studies, 34(2),

231-270. https://doi.org/10.7202/1072652ar viewed online.

https://apropos.erudit.org/en/users/policy-on-use/ 


\section{Étude du risque dans la vie maritime autour de Saint-Pierre-et-Miquelon 1817-1948}

Nicolas Landry

Cette étude s'inscrit dans une longue tradition historiographique portant sur les risques de la vie maritime, autant dans le commerce que dans la pêche et ce, à l'époque de la voile et de la vapeur durant le 19 siècle et la première partie du $20^{\mathrm{e}}$. Après avoir dressé un bilan historiographique et méthodologique sur les tendances actuelles en études des naufrages et des pertes de vie en mer, je présente les résultats d'une recherche préliminaire à partir des données recueillies dans le registre des marins disparus et décédés en mer, qui se trouve au musée l'Arche à Saint-Pierre-et-Miquelon. Cette source permet de brosser un portrait de la population à l'étude. Dans une perspective plus large, précisons que cette recherche fait partie du projet de recherche Archipel, à partir duquel j'ai déjà publié quelques articles ${ }^{1}$. Jusqu'à maintenant, je me suis surtout intéressé à des démarches de nature démographique et économique portant sur la période 1763-1920.

\section{Cadre historiographique et méthodologique}

Les repères historiographiques servant à contextualiser cette recherche sont multiples. Il semblerait qu'en France, le 19e siècle est demeuré pendant longtemps une époque « peu explorée » en ce qui concerne les "populations maritimes » ${ }^{2}$. Toutefois, un sérieux coup de barre est donné à partir des années 1980 pour mieux faire connaître les réalités de ceux qu'on appelait gens de mer. Cette introduction historiographique fait d'ailleurs amplement référence aux travaux de quelques-uns des 
chercheurs les plus prolifiques de la thématique maritime du $19^{\mathrm{e}}$ siècle. Il suffit de mentionner Gérard Lebouëdec, Alain Cabantous ou encore Thierry Sauzeau.

Les travaux portant sur les risques de la vie maritime font ici fonction de cadre référentiel aux risques vécus par les marins-pêcheurs métropolitains et de l'Archipel, participant à la grande pêche de TerreNeuve. Lobjectif de cette recherche demeure avant tout centré sur les risques et les fatalités auxquels plusieurs de ces hommes font face ${ }^{3}$, incluant les pêcheurs résidents de Saint-Pierre-et-Miquelon. À l'instar des travaux de Brian Payne, il y a donc volonté de comparer des histoires locales d'une région frontière (côte sud-ouest de Terre-Neuve et Saint-Pierre-et-Miquelon) qu'est le golfe du Saint-Laurent et plus précisément ce voisinage franco-britannique. Nous estimons qu'il s'y partage alors des sensibilités communes ${ }^{4}$.

Suivant cette perspective, il est pertinent de s'interroger sur la représentation que l'on se fait du pêcheur au $19^{\mathrm{e}}$ siècle, du moins aux yeux d'un visiteur français de l'époque comme l'était Arthur Gobineau? Il était d'avis que ces hommes de la mer « sont une apparition d'un autre âge et à coup sûr d'une autre société ». Ils sont des êtres " intrépides et indépendants, remarquables par leur vigueur et leur énergie physique ${ }^{5}$. Cependant, Jean-Michel Auffray estime difficile de circonscrire une véritable « définition du marin ». Un grand nombre d'entre eux sont souvent de « jeunes paysans se métamorphosant en pêcheurs et en marins» tentant de tirer profit des campagnes de pêche à Terre-Neuve ou en Islandé .

Dans un premier temps, qu'en est-il de la situation économique de ces gens de mer en France, à savoir s'ils choisissent volontairement ou non de s'embarquer? L'historiographie a déjà établi, depuis un bon moment, que ces marins préconisent une approche de pluriactivité économique afin de renflouer les coffres de la famille ${ }^{7}$. Ils pratiquent la grande pêche mais aussi le commerce et sont pour la plupart inscrits sur le registre des matricules de la Marine ${ }^{8}$. C'est d'abord l'expérience qui permet d'avancer dans la carrière de matelot et non la formation ${ }^{9}$. Du moins jusqu'au début du $20^{e}$ siècle. On est d'abord mousse avant 
de gravir les échelons spécialisés de la grande pêche ${ }^{10}$. Par exemple, jusqu'au « décret de 1852 », celui détenant le titre "d'officier à la grande pêche " ne détient aucun diplôme spécifique ${ }^{11}$. De manière générale, ces marins voient leur espérance de vie fondre d'une dizaine d'années en moyenne. Au $18^{\mathrm{e}}$ siècle, la " mortalité océane prend un homme sur deux avant l'âge de 35 ans $»^{12}$. Dans les navires de pêche partant de Dunkerque entre 1835 et 1839, 404 hommes disparaissent dont $7 \%$ sont pêcheurs ${ }^{13}$. Les autres pertes de vies sont attribuables aux décès à bord en raison d'accidents, de maladie, certains marins passent par-dessus bord alors que d'autres décèdent dans les ports. Il y a également des augmentations de mortalité entre 1880 et 1900, dans certaines communautés côtières de la façade atlantique en France ${ }^{14}$. Globalement, Sauzeau estime que cette «vie maritime française » se distingue par une « forte mortalité professionnelle $»^{15}$.

Dans un deuxième temps, notons que les marins du $19^{\mathrm{e}}$ siècle sont témoins de trois phénomènes qui leur sont plutôt favorables soit l'industrialisation de la navigation, une amélioration de la sécurité maritime et de meilleurs soins de santé. Contrairement aux préjugés propagés au $19^{\mathrm{e}}$ siècle, les marins-pêcheurs « s'adaptent à de nombreux changements techniques, économiques et structuraux $»^{16}$. La littérature récente fait ressortir que la navigation à voile du $19^{\mathrm{e}}$ siècle amorce une longue phase, de déclin pour certains mais de transition pour d'autres, avec l'industrialisation et la vapeur. Ces changements économiques et technologiques semblent toutefois augmenter les exigences sur les navires et envers les matelots, puisqu'on tente d'augmenter la vitesse des navires et la productivité à bord ${ }^{17}$. C'est aussi une époque où se manifestent et se précisent certaines règles relatives aux sauvetages, aux naufrages et aux collisions. Des chercheurs ont statué que les succès d'un voyage commercial reposent sur plusieurs facteurs incluant la quantité et la qualité des provisions, de même que sur l'état d'esprit et la compétence des équipages et des officiers ${ }^{18}$. Un bon équipage doit pouvoir s'ajuster au gréement qui peut bouger selon la vitesse des vents, les conditions de la mer, les changements de direction du navire, ou l'état des voiles ${ }^{19}$. 
En dépit des nouveaux défis de la navigation du $19^{\mathrm{e}}$ siècle, les progrès technologiques contribuent donc à une certaine amélioration des conditions de travail des marins. D'abord, il y a une « baisse de la durée des trajets en mer, une meilleure hygiène à bord, un recul du typhus et du scorbut. Même qu'il y aurait une augmentation de l'espérance de vie des marins et ceux atteignant 51 ans accèdent à une pension de retraite $»^{20}$. Effectivement, Patrick Louvier estime qu' « entre le $18^{\mathrm{e}}$ et la fin du premier $19^{\mathrm{e}}$ siècle, le taux de mortalité baisse lentement grâce à l'accumulation de progrès fondamentaux $»^{21}$. Parmi les innovations technologiques significatives, mentionnons l'utilisation du fer dans la construction navale et la vapeur comme moyen de propulsion. Après 1869, l'hélice remplace la roue à aubes, en 1904 c'est l'apparition de la première goélette frigorifique et en 1905 arrive le chalutier à vapeur. Il n'en demeure pas moins qu'entre 1840 et 1860, les équipages souffrent encore de la présence d'une grande «saleté, de la promiscuité, des fièvres typhoïdes, d'épidémies et d'accidents de travail $»^{22}$. C'est d'abord dans la marine de guerre que les choses s'améliorent à compter de 1885 et ce, dans le domaine des soins de santé. On note alors des " avancés cliniques et thérapeutiques $»^{23}$.

On pense ici à l'avènement des hôpitaux en milieux maritime, de la Société des Euvres de mer de France et des lazarets ${ }^{24}$. Du côté britannique durant les années 1860, comme en France, la santé des marins de la marine marchande est très inférieure à celle des matelots de la Royal Navy. Par exemple, si le scorbut disparait des navires de la Navy à compter des années 1790, il se manifeste encore dans la marine marchande durant les années 1850. Il est toutefois permis de croire que l'adoption du Merchant Shipping Act de 1867 resserre les exigences visant à éradiquer la maladie, à fournir une meilleure alimentation et un milieu de vie plus sain. Il semble que des progrès dans la construction des navires, durant la Première Guerre mondiale, contribuent aussi à un meilleur milieu de vie des équipages ${ }^{25}$.

Des préoccupations envers le bien-être des pêcheurs et leurs familles se manifestent à la même époque. Par exemple, durant lère victorienne en Grande-Bretagne, un organisme de charité maritime est 
fondé en 1839. Chapeauté par un comité central à Londres, il est représenté par des agents locaux dans les régions côtières, notamment en Écosse, où les communautés de pêcheurs sont secouées par le Moray Firth Disaster de 1848. Cette tempête cause la perte de 124 bateaux, la mort d'une centaine de pêcheurs, laissant 47 veuves et 161 enfants $^{26}$, à qui l'on fournit des secours de première nécessité. La Société des Cuvres de mer est fondée en France en 1894 pour fournir une « aide matérielle et morale aux marins terre-neuvas ». À ce moment-là, on estime à 15000 le nombre d'hommes impliqués dans la grande pêche se déroulant au large de l'Islande et sur les bancs de Terre-Neuve. On met alors de l'avant les dangers guettant les pêcheurs; " perte de doris dans la brume, abordages, tempêtes, épuisement, hygiène déplorable ». Les responsables de la SOM ciblent aussi « trois fléaux de la grande pêche » soit "l'alcoolisme, la maladie et l'isolement " ${ }^{27}$. Précisons que c'est à compter de 1898 que la SOM arme deux navires (le SaintPierre et le Saint-Paul) pour accompagner la flotte des morutiers au large de l'Archipel ${ }^{28}$. De 1896 à 1939, soit environ 39 campagnes de pêche, les navires de la SOM fournissent un total de « 32000 journées d'hôpital, 132000 consultations médicales et réussissent à recueillir en vie 426 victimes de naufrages ». À cela s'ajoutent des « maisons de famille » à Saint-Pierre-et-Miquelon pour les pêcheurs en « escale ou en convalescence ${ }^{29}$.

Si des actions sont mises de l'avant pour améliorer la santé des marins-pêcheurs, quen est-il de l'évolution de la sécurité dans la navigation au $19^{\mathrm{e}}$ siècle? Le chercheur peut d'abord consulter les fameux routiers de Martin de Hoyarsabal et de Piarres Detcheverry, datant respectivement du $16^{\text {e }}$ et du $17^{\mathrm{e}}$ siècles. On y trouve des cartes remplies d'indications permettant de suivre les routes de navigation sur les Grands Bancs et autour de Terre-Neuve, à l'aide de points de repères. Ces deux ouvrages deviennent donc fort appropriés lorsqu'on se réfère à Saint-Pierre-et-Miquelon ${ }^{30}$.

Pour mieux saisir les éléments essentiels au contexte de la grande pêche, il ne faut pas limiter l'étude à la situation en mer $^{31}$ mais s'intéresser aussi à la problématique des dangers des littoraux. Bryn Tapper 
aborde cette question dans son étude sur le French Shore de TerreNeuve. Cet auteur met de l'avant le concept de « Maritime Cultural Landscape ", dans lequel il intègre la relation entre des points de repère et les lieux où se déroulent les activités humaines. Il estime qu'avec le temps un réseau de repères se serait élaboré ou construit, formant des routes maritimes et des lieux d'ancrage ${ }^{32}$. Ce réseau permet aux marins d'éviter les dangers submergés tels les hauts fonds et les rochers. Vers 1821, 216 ports de pêche sont en opération sur le French Shore et jusqu'en 1872, le nombre d'embarcations varie entre 2205 et $2172^{33}$.

À ces points de repères s'ajoutent les connaissances de navigation des marins et celles des relevés topographiques de la Marine française, effectués entre 1827 et 1864. Selon Korsgaard et Gibbs, les aides à la navigation seraient de deux ordres soit celles de nature artificielle (bouées, phares, marques) soit celles plutôt naturelles (grands rochers, arbres) $)^{34}$.

Mais, tel que mentionné plus haut, ces évolutions n'empêchent pas les accidents maritimes de se produire tout au long du $19^{\mathrm{e}}$ siècle dans le territoire à l'étude. Ce constat devient évident si l'on s'intéresse au retour de la grande pêche française sur les bancs de Terre-Neuve et dans le golfe du Saint-Laurent à compter de 1815. Rappelons que la fin des guerres napoléoniennes et impériales entre la France et l'Angleterre, permet le rétablissement de la France à Saint-Pierre-etMiquelon. Des armateurs de Fortrieux, Binic, Légué, « reconstituent leurs flottes de bricks, de trois mâts ». À Phéneuf, la majorité des inscrits maritimes participent à la grande pêche ${ }^{35}$. Les pêcheurs français profitent aussi d'une prime instaurée en 1816 et cet incitatif fonctionne puisque de 160 navires à Terre-Neuve en 1820, leur nombre atteint 420 en 1830, employant près de 12000 hommes. Le rythme se maintient jusqu'en 1850. Du côté de Saint-Pierre-et-Miquelon, la morue débarquée est destinée à être séchée, autant celle des résidents que des pêcheurs saisonniers ${ }^{36}$.

Ainsi, durant la première moitié du $19^{\mathrm{e}}$ siècle, les armements morutiers de Bretagne sont prospères puisque des revenus substantiels proviennent de l'exportation du poisson en Méditerranée ${ }^{37}$. Il 
semblerait toutefois que les choses changent à partir de 1860, entre autres dans les armements de Saint-Brieuc. Des difficultés surgissent à Terre-Neuve puisque la morue devient plus rare et l'on constate qu'il sétablit une certaine opposition coloniale anglaise envers la présence française ${ }^{38}$. Mais Fournet, lui, estime que des changements se manifestent durant toute la période à l'étude (1816-1904) alors qu'il y a bel et bien "transition de la pêche sédentaire à la pêche errante " ${ }^{39}$. Rappelons que cette dernière se pratique au large sur les bancs par des goélettes de 100 tonneaux, originaires de Saint-Pierre, mais surtout par des trois ou quatre mâts de 400 à 500 tonneaux armés dans un petit nombre de ports métropolitains (Fécamp, Saint-Malo, Paimpol, Granville, Cancale et La Rochelle). En 1900, près de 240 navires se livrent à ce type de pêche. La morue est pêchée avec des lignes de fond ${ }^{40}$ relevées chaque matin par des doris ${ }^{41}$, montés par deux hommes. Le poisson, préparé et salé à bord, reste dans la cale du navire mère jusqu'à la fin de la campagne ${ }^{42}$. Pour la pêche côtière, Tapper parle de chaloupes mesurant de 6 à 10 mètres, montées par trois hommes avec chacune deux graviers qui manutentionnent leur morue. Ces embarcations s'éloignent jusqu'à un mille de la côte, partant le matin et revenant en fin de journée ${ }^{43}$.

Au terme de ce survol historiographique, nous connaissons mieux certaines composantes de l'univers maritime des pêcheurs. S'il demeure fort difficile d'établir si leur statut socio-économique s'améliore, certaines innovations technologiques marquent la période à l'étude. Les traversées deviennent moins longues et plus sécuritaires, notamment en raison de l'avènement progressif des navires à vapeur et d'infrastructures de santé. C'est ainsi que de meilleurs soins peuvent être prodigués à bord, sur un navire hôpital ou dans un hôpital de port, entre autres à Saint-Pierre. Mais en dépit de cela, les accidents maritimes demeurent fréquents et assujettis aux tempêtes, aux accidents et à la maladie à bord ou même dans l'Archipel. La section suivante permet justement de mieux saisir le contexte des accidents maritimes. 


\section{Contexte théorique pour l'étude des naufrages}

Durant la première moitié du $19^{\mathrm{e}}$ siècle, les statistiques sur les naufrages ont probablement de quoi inquiéter les autorités britanniques. En effet, $5 \%$ des vaisseaux qui naviguent vers les Indes occidentales périssent dans les naufrages, surtout en raison du mauvais temps. En 1830, on estime à 100 par année les pertes de vie résultant de ces naufrages ${ }^{44}$. Du côté de certaines communautés côtières françaises, on déplore là aussi de nombreuses pertes de vie. Par exemple, dans la chapelle Notre-Dame-des-Auzils de Gruissan, se trouvent des cénotaphes des XIXe et XXe siècles commémorant les morts en mer. Les tombeaux vides représenteraient un genre de " cimetière marin » rappelant la « vocation maritime de la cité $»^{45}$.

Les chercheurs Korsgaard et Gibbs proposent une série de questions permettant d'établir des modèles et tendances des naufrages; les caractéristiques techniques des vaisseaux, les causes d'accidents maritimes et leur distribution dans l'espace et dans le temps, de même que les pertes de vie ${ }^{46}$. Ces chercheurs ont entre autres constaté que plusieurs naufrages surviennent à proximité de lieux côtiers où l'on trouve de l'eau douce, du bois, des produits alimentaires frais et des lieux d'ancrage $^{47}$. À l'instar de mes résultats de recherche, ces auteurs observent que certaines années sont plus propices aux naufrages que d'autres, surtout en raison de la météo. Effectivement, on constatera plus loin dans le texte que certaines années sont plus fertiles en tempêtes. Parmi les autres causes de nature environnementale c'est souvent la présence d'un récif, d'une météo imprévisible ou encore de courants changeants $^{48}$. Quant à Duncan et Gibbs, ils ont dressé un schéma des étapes d'un naufrage; pré-contact, perception d'un danger, avertissement, impact, danger de pertes de vie, sauvetage, choc post-traumatique attribuable à l'événement ${ }^{49}$.

Mais avant d'aborder la question des accidents maritimes, présentons un survol de l'évolution des pêches à Saint-Pierre-et-Miquelon durant la période à l'étude. Il s'agit d'une mise au point importante à faire puisque l'Archipel abrite non seulement une population vivant 
d'abord et avant tout de la pêche, mais qui se situe aussi aux premières loges pour observer et vivre un bon nombre de tragédies frappant les pêcheurs autant métropolitains que de l'Archipel.

\section{Le cas de Saint-Pierre-et-Miquelon}

L'archipel connaît un véritable « âge d'or » de la pêche entre 1816 et 1910 et ce, en dépit des grands incendies de 1844,1865, 1867 et 1902. Marc Pabois pense toutefois que cette étiquette s'adresse surtout aux armateurs français et aux commerçants de Saint-Pierre. Le schéma spatial de l'archipel durant la période à l'étude se compose d'espaces d'échouage, de salines, de graves, de maisons et de jardins ${ }^{50}$. Aux îles, la pêche à la morue se déroule de mai à septembre et ce, dans un rayon d'une dizaine de milles autour de l'archipel. L'essentiel des activités de pêche prennent place « au sud et sud-est de l'île Saint-Pierre, dans les parages de l'île Verte, dans la Baie, au sud-est du Cap Miquelon et à l'ouest de la Grande Miquelon, en particulier au large de la région comprise entre la Pointe au Cheval et le Cap Blanc » ${ }^{51}$.

Sanguin explique que l'archipel est formé de « cinq ensembles soit Saint-Pierre, Langlade, la dune de Langlade, Miquelon et la presqu'île $\mathrm{du}$ Cap $~_{52}$. À l'̂̂le-aux-Marins, on compte une cinquantaine de familles et une église. Il semblerait que les pêcheurs de cette communauté soient toujours les premiers à s'embarquer pour la pêche le jour même de la bénédiction de la mer. Selon une tradition basque, cet événement se déroule le jour de la Trinité soit entre la mi-mai et la mi-juin ${ }^{53}$. Il s'agit en somme d'une fête à date mobile. L'île Miquelon, elle, est « peuplée de 500 habitants avec de modestes demeures entourées d'un jardinet cultivé » ${ }^{54}$.

Si l'on revient à l'hypothèse de la période d'âge d'or (1816-1910), les chiffres tendent à le démontrer et ce, autant du point de vue démographique que du nombre de goélettes et de la productivité. D’abord, la population passe de 600 à 6842 personnes entre 1816 et 1902. Et cela n'inclue pas les 4000 graviers bretons, normands, basques et même terre-neuviens employés durant la saison de pêche. Toutefois, 
entre 1903 et 1908, une crise économique aurait provoqué le départ de 2500 personnes vers le Canada ${ }^{55}$. On impute ce phénomène à de mauvaises campagnes de pêche et à l'assujettissement aux armements métropolitains. Alors que les années 1920 font place à une certaine reprise grâce à la prohibition américaine, les années 1940 donnent lieu à une véritable industrialisation. Ainsi, près de 500 chalutiers fréquentent les Grands Bancs de Terre-Neuve chaque année et Saint-Pierre en bénéficie à titre de port d'escale ${ }^{56}$. Toutefois, la population est assez stable entre 1911 et 1941, passant de 4209 à $4425^{57}$. Il faut attendre jusqu'en 1999 pour voir la population remonter au-delà des 6000 personnes $^{58}$.

Pour revenir à la pêche, notons que durant le $19^{\mathrm{e}}$ siècle il arrive de France des « dizaines de navires » et de 1500 à 1800 graviers qui s'activent à la préparation du poisson. D'autres ouvriers construisent des " hangars, des magasins, aménagent des graves et des routes ${ }^{59}$. Des maisons de commerce métropolitain sont également représentées dans l'archipel par des « agents d'habitation $»^{60}$. Ce type d'infrastructure comporte le logement du gérant, des magasins (hangars), des espaces de prairies « recouvertes d'un lit de pierres blanches (on peut en douter?) où faire sécher la morue $»^{61}$. Le nombre de goélettes locales, lui, passe de la vingtaine en 1825 à 284 en $1903^{62}$. À Miquelon, on pêche dans le golfe du Saint-Laurent et autour des îles de la Madeleine sur des goélettes de 10 à 15 tonneaux. Ce poisson est rapporté pour être séché à Miquelon avant d'être vendu à Saint-Pierre ${ }^{63}$.

Jusqu'au début du $20^{\mathrm{e}}$ siècle et l'avènement des chalutiers, les bâtiments armés pour la pêche à la morue sont équipés de doris, dont on a déjà parlé, et de lignes dormantes. Chaque jour, le marin-pêcheur de doris doit « boëtter plusieurs milliers d'hameçons et aller mouiller les lignes $»^{64}$. Mais cet âge d'or entame un long déclin avec l'arrivée des premiers chalutiers à vapeur durant la Première Guerre mondiale. Ces bâtiments sont " grands, autonomes et laissent peu de place aux armateurs locaux $»^{65}$. L'impact est frappant puisque le nombre de goélettes de l'archipel passe de 284 à 25 entre 1903 et 1914, et à seulement deux en $1919^{66}$. Toutefois, les chiffres d'Ancelin, eux, laissent plutôt penser à un déclin moins rapide que semble le croire Sanguin et De Curton. 
Ancelin estime plutôt que de 1880 à 1910, le nombre de goélettes en opération dans l'archipel passe de 142 à 44 et celui des doris de 247 à $384^{67}$. C'est donc une hausse pour ces derniers ${ }^{68}$. Il est ici approprié de se demander si l'augmentation du nombre de doris ne résulte pas d'une « réorientation pour les pêcheurs locaux »? En effet, si le nombre de goélettes diminue, les pêcheurs se réorientent davantage vers la petite pêche côtière ${ }^{69}$. À ce stade de ma recherche, il n'est pas possible de savoir si ce changement serait perceptible dans les données du registre des marins disparus et décédés .

Pour revenir au propos de cette recherche, soit les marins disparus et décédés, les auteurs cités ci-dessus soulèvent tous les dangers des métiers de la mer et les pertes matérielles et humaines qui en résultent. D'abord, Sanguin estime qu'entre 1816 et 1984, au moins « 650 naufrages » surviennent sur les côtes de l'archipel dont 95 sur la dune de Langlade, la " nécropole des navires ». Les causes de ces pertes, déjà mentionnées dans ce texte, sont « les vents, les courants, la neige et la brume $»^{70}$. Quant à Ancelin, il estime à « 96 naufrages en quatre ans » les pertes subies durant les années $1860^{71}$. Finalement, pour De Curton, « la dune de sable reliant Miquelon à Langlade est un véritable cimetière de navires $»^{72}$.

C'est donc à cette étape de ma recherche qu'il faut tenir compte des données du registre des marins disparus et décédés, mais en limitant pour l'instant mes observations à Saint-Pierre-et-Miquelon. Dans un premier temps, j'ai trouvé intéressant de classer les victimes des accidents maritimes selon leurs lieux de naissance ou encore de résidence dans l'archipel, au moment de l'accident. Ainsi, pour 248 cas présentant ce genre d'information, 185 (65\%) sont associés à SaintPierre et 58 (40\%) à Miquelon. Il est tout aussi intéressant d'identifier les types d'embarcations impliquées et le nombre d'hommes associés à chaque type. Il s'agirait donc de 81 goélettes auxquelles sont associés 150 hommes soit $76 \%$ du nombre total d'hommes touchés par des accidents maritimes dans l'archipel. Il est ensuite possible de reconstituer les équipages pour six de ces goélettes soit la Madelaine (10 hommes), la LMB (8 hommes), la Gentilla (8 hommes), la Blanche 
(6 hommes), l'Ali Baba (6 hommes) et la Paul et Louis (6 hommes). Au total, 44 hommes ayant monté l'une de ces goélettes à un moment ou à un autre, a vécu un accident maritime quelconque. Pour leurs voisins de la côte de Terre-Neuve, je relève 40 victimes d'accidents maritimes. Pensons entres autres à Victor Audoux, né à Saint-Georges en 1845, mais inscrit à titre de matelot de $3^{\text {e }}$ classe à Saint-Pierre. La section suivante, elle, permet d'en apprendre davantage sur les circonstances de certains accidents maritimes survenus dans l'archipel durant la période à l'étude.

\section{Narration des accidents maritimes dans l'archipel, à partir du registre des marins disparus et décédés}

$\mathrm{Au}$ même titre que les victimes d'accidents maritimes mentionnés dans la section Le registre des marins disparus et décédés, celles de l'archipel ont aussi leurs histoires à raconter. Par exemple, la plus ancienne mention d'accident maritime du $19^{\mathrm{e}}$ siècle pour Miquelon est celle de la disparition de Jean-Édouard Poirier, en date du 26 octobre 1817. ${ }^{73}$. Mais si l'on s'en tient à l'essentiel, mentionnons que deux grandes tempêtes affectent au moins 37 hommes de l'archipel soit celles du 25 août 1873 (23) et celle du 13 septembre 1900 (14). Dans le premier cas, les frères Édouard-Antoine et Pierre Théophile Enguehard, de Miquelon, disparaissent simultanément sur les goélettes Brunette et Jeanne-Marie Joseph ${ }^{74}$. Ils sont les fils de Jean-Baptiste et d'Aimée Poirier et respectivement âgés de 27 et de 38 ans. Ensuite, lors de la tempête de 1900, Eugène Joseph Frioult, 20 ans, disparait et son cadavre est retrouvé à West Point sur la côte de Terre-Neuve. La goélette qu'il montait, Fio$n a$, est alors retrouvée « chavirée en mer $»^{75}$. Mais pire encore, les frères Xavier Yves et Joseph Victor Lafourcade se noient ensemble à bord de la goélette $A l i B a b a^{76}$. L'histoire d'Henri Gratien Jugan est encore plus particulière et peut-être empreinte d'un certain fatalisme puisqu'il perd la vie en juin 1908, en participant au sauvetage du Marie-Thérèse à Savoyard. Son corps est retrouvé le lendemain ${ }^{77}$.

Mais d'autres aspects sont à retenir dans l'étude des accidents 
maritimes de l'archipel. Mentionnons d'abord les délais survenant entre le signalement d'une disparition et le recouvrement du défunt. Par exemple, George Bonnel, de Lamaline (Terre-Neuve), disparait au début mai 1891 et son cadavre n'est retrouvé que le 5 juillet ${ }^{78}$. L'un de ses compatriotes, Georges Himberton, de Burin, est déclaré disparu à Barachois (Saint-Pierre) à la fin d'avril 1884. Mais le 5 juin de la même année, un cadavre « inconnu supposé être le sien » est découvert ${ }^{79}$.

Toutefois, certaines situations ne semblent n'avoir rien à voir avec des accidents à caractère maritime, incluant de nombreux cas de terre-neuviens. Rappelons celui de James Cusick, de Saint Lawrence (Terre-Neuve), retrouvé mort dans la rue Jacques Cartier à SaintPierre ${ }^{80}$. C'est un scénario à peu près similaire pour d'autres victimes dont Thimoté Martin, de Burin, retrouvé près du ruisseau Courval sur l'île Saint-Pierre en avril $1856^{81}$, ou encore Dominique Dacosta de Baie Saint-Georges (Terre-Neuve), décédé à l'Hôpital de Saint-Pierre en octobre 1878 à 19 ans seulement. Toutefois, lui non plus ne semble pas être victime d'un accident maritime. Il avait auparavant été condamné à un an de prison et se trouvait à l'hôpital pour y recevoir des soins $^{82}$. S'ajoutent à ces décès dans des circonstances vaguement maritimes, ceux de Pierre Jean-Baptiste Lelorieux, de Baie Saint-Georges en 1871 à l'Anse à Ross (Langlade) dans un accident de chasse au gibier de mer $^{83}$, Jean Toben de Saint-Laurent au Lazaret de Savoyard en $1886^{84}$ et finalement, Edouard Turpin, également de Saint-Laurent, au domicile de François Saillard, de Saint-Pierre, en 1876 ${ }^{85}$. Tel qu'évoqué ailleurs dans ce texte, peut-être y était-il employé?

Il est ici permis de constater qu'en dépit d'une période dite d'âge d'or de la pêche dans l'archipel, les pêcheurs du lieu ne sont guère épargnés par les accidents maritimes. Tout comme ceux de la métropole, ils sont victimes de soudaines levées de brouillard ou de vents forts pouvant les faire dériver, se perdre ou se noyer. Par tempête, d'autres vont aussi s'échouer sur la côte ou devoir affronter une collision parfois fatale avec des récifs à fleur d'eau ou des rochers. Ces tragédies ne sont pas seulement consignées dans le registre des marins disparus et décédés, mais aussi dans La Feuille officielle de l'archipel ou dans 
d'autre journaux publiés entre 1866 et 1896 dont l'Égalité, Le Patriote ou encore l'Impromptue $e^{86}$. On y trouve à la fois les circonstances des accidents et les noms des victimes. On peut se consoler en pensant que les familles de l'archipel ont davantage de chances que ceux de la métropole de récupérer leurs morts pour être en mesure de célébrer des funérailles convenables. Malgré les années qui passent, le souvenir de ces disparus est préservé.

\section{Le registre des marins disparus et décédés}

Rappelons que le registre des marins disparus et décédés inclut autant ceux disparus ou morts en mer qu'ailleurs sur les littoraux étudiés ${ }^{87}$. Je répète qu'il ne s'agit pas d'une étude sur les naufrages, mais plutôt sur les victimes soit les marins, les pêcheurs et les passagers. Notons également que je privilégie le terme d'accidents maritimes plutôt que ceux de noyade ou de décès. Du moins pour la sous-catégorie de ceux dits "disparus en mer ", versus les décès officiels survenus à bord des navires, le long du littoral, à terre ou dans un hôpital. Qui plus est, plus loin dans cette étude, je propose des chiffres sur le nombre de disparus repêchés. On a donc un nombre total de 6448 cas observables dont 2199 décès confirmés (hôpitaux de Saint-Pierre et décès à bord des navires de toutes sortes), soit $34 \%$ du nombre de cas observés. On peut donc en conclure qu'un marin en situation de risque d'accident maritime, a environ $66 \%$ de chance de s'en tirer.

Toutefois, en raison des délais survenant entre le moment du constat d'une disparition et la disponibilité d'information sur le sort d'une victime, il devient difficile de statuer de manière certaine sur le sort final et officiel de ces victimes d'accidents maritimes. Certains de ces hommes supposés perdus, peuvent parfois réapparaitre des semaines plus tard pour reprendre du service. Également, pour les années où il n'y a aucune déclaration de décès ou de disparition, est-ce bien le cas ou s'agit-il simplement d'une lacune dans le système d'enregistrement? Par exemple, le petit nombre d'accidents maritimes s'explique pour les périodes des deux guerres mondiales (1914-18 et 1939-45) puisque la 
pêche française était alors pratiquement en suspens.

La liste portant sur les marins décédés ou disparus en mer a donc permis de dresser un inventaire total de 6448 cas observables, mais pour lesquels la complémentarité de l'information a tendance à varier $^{88}$. D'abord, dans le cas des décès à l'hôpital ${ }^{89}$, la majorité (44\%) des 1463 cas se concentrent sur 24 années où l'on rapporte 20 décès ou plus soit un total de 648. Sept années s'avèrent particulièrement funestes soit 1903 (44), 1902 (37), 1885 (36), 1886 (36), 1900 (35), 1894 (30) et 1893 (30). Ces hommes mourant pour la plupart très loin de chez eux, sont victimes de maladies contractées lors des traversées ou de blessures de travail subies à bord ou à terre. À noter qu'un petit nombre d'hommes, surtout des hivernants, meurt parfois chez l'habitant et j'y reviens plus loin dans le texte. En ce qui a trait aux 736 décès à bord, ils séchelonnent entre 1828 et 1949 . Le plus souvent, ils surviennent durant les traversées, résultats de maladies ou d'accidents de travail en mer. On note dix années avec 20 décès ou plus soit 255 ou $34 \%$ du total. Les trois années les plus funestes sont 1902 (37), 1903 (36) et 1900 (31).

Tel quévoqué au début de ce texte, la catégorie des disparus en mer rend difficile un calcul des décès définitifs. C'est pourquoi je préfère parler de victimes d'accidents maritimes pour ces hommes, dont le destin n'est pas toujours retraçable. Quoiqu'il en soit, les chiffres à cet égard révèlent un total de 4249 constatations de disparition en mer entre 1817 et 1948 soit 121 années observables. On parle donc ici d'une moyenne annuelle de 35 disparitions. Certaines années s'avèrent particulièrement sinistres avec un total de 983 cas ou $23 \%$ du total des rapports de disparition. Ces années sont 1873 (250), 1900 (213), 1892 (146), 1894 (130), 1883 et 1891 avec 122 dans les deux cas. Ces années coïncident souvent avec des tempêtes particulièrement violentes ${ }^{90}$.

À titre d'analyse d'échantillon, j'ai choisi d'examiner l'âge moyen des disparus pour 87 hommes dont les noms de famille figurent à la lettre A dans la liste. Il en résulte un âge moyen de 39 ans, quoique 19 d'entre eux ont 20 ans ou moins soit $22 \%$ du total. Par exemple, signalons Pierre Auguste, 14 ans, né à Saint-Malo, novice, qui 
s'embarque à Dinan, où il est classé. Il meurt à l'hôpital de Saint-Pierre le 17 septembre $1855^{91}$. À l'autre bout du spectre, Laurent Aubert, 68 ans, né à St-Pair en 1813, marin, disparu en mer le 13 décembre 1881 et dont le corps est retrouvé au Cap-Rouge de Saint-Pierre. Il est utile de préciser que les exemples de ce genre sont légion.

Il est tout aussi intéressant de connaître les ports d'origine des marins affectés par ces accidents maritimes, funestes ou non. On parle ici des ports d'enregistrement des classes. C'est ainsi que j'en arrive à un total de 5564 identifications. Toutefois, ces ports se subdivisent en catégories d'importance décroissante. Les trois principales catégories de ports s'approprient un total de 5419 déclarations. Je les qualifie de ports prédominants (six pour $4568 \mathrm{cas}$ ), ports secondaires (cinq pour 665 cas) et ports marginaux (six pour $186 \mathrm{cas}$ ). Le port le plus important est Dinan avec 1440 déclarations, soit 26\% du total de 5564 . Il est suivi de Cancale avec 900 et de Saint-Malo avec 743. La majorité des marins se sont donc embarqués dans trois ports soit Dinan, Cancale et Saint-Malo, situés dans un rayon de moins de 30 kilomètres. À noter qu'au lendemain de la Première Guerre mondiale, des ports réputés très actifs jusque-là, tels Granville, Paimpol et La Rochelle, s'éclipsent peu à peu au profit de Fécamp, Saint-Malo et Bordeaux ${ }^{92}$.

Tableau 1: Compilation du nombre de marins inscrits au matricule et victimes d'accidents maritimes par ports d'embarquement

\begin{tabular}{|c|c|c|c|c|c|}
\hline $\begin{array}{c}\text { Ports } \\
\text { prédominants }\end{array}$ & Hommes & $\begin{array}{c}\text { Ports } \\
\text { secondaires }\end{array}$ & Hommes & $\begin{array}{c}\text { Ports } \\
\text { marginaux }\end{array}$ & Hommes \\
\hline Dinan & 1440 & Fécamp & 177 & Regneville & 46 \\
\hline Cancale & 900 & Binic & 155 & Bayonne & 41 \\
\hline St-Malo & 743 & Lannion & 127 & Dieppe & 33 \\
\hline Paimpol & 617 & Tréguier & 109 & $\begin{array}{c}\text { St-Valéry en } \\
\text { Caux }\end{array}$ & 24 \\
\hline St-Brieuc & 469 & $\begin{array}{c}\text { St-Jean-de- } \\
\text { Lux }\end{array}$ & 97 & Morlaix & 23 \\
\hline Granville & 399 & & & Brest & 19 \\
\hline Total & 4568 & & 665 & & 186 \\
\hline
\end{tabular}




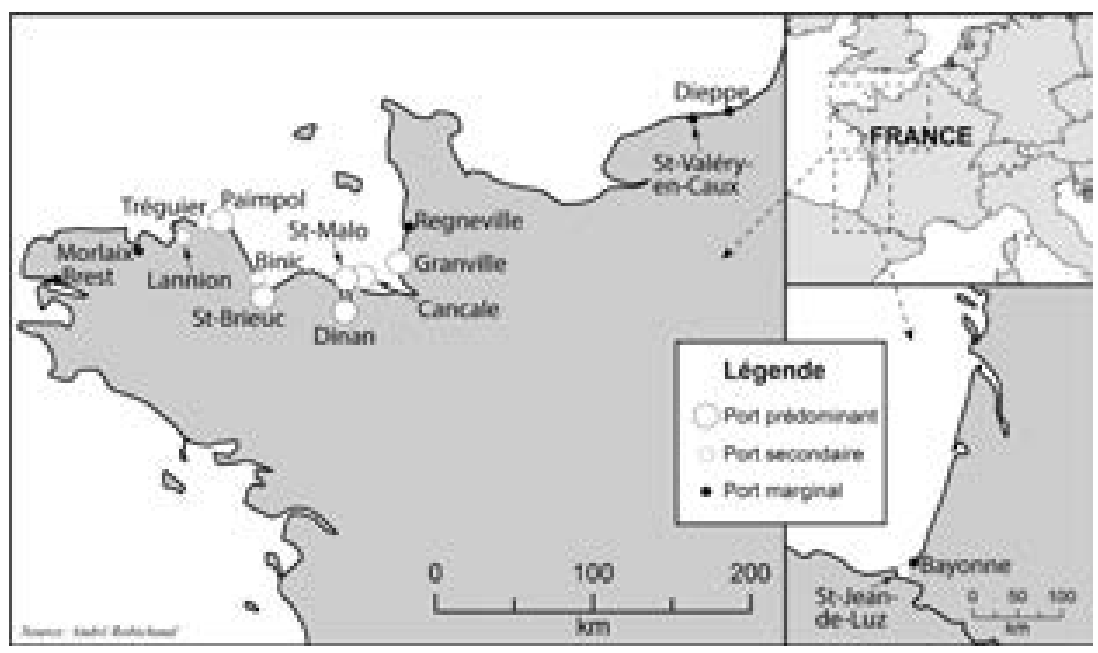

Figure 1. Cette carte illustre le territoire où se situent la majorité des ports figurant au tableau 1. Elle a été produite par le professeur André Robichaud de I'Université de Moncton au campus de Shippagan.

Les efforts de compilation globale deviennent plus complexes lorsque vient le temps de brosser un portrait des catégories professionnelles ou classement de chaque homme. Pour clarifier la question, je sépare le statut d'inscription de celui de la fonction exercée dans les activités de pêche. Dans un premier temps, j'arrive à neuf catégories de matricules pour un total de 5856 hommes classés. Ce sont les matelots et les matelots dits de $3^{\mathrm{e}}$ classe qui, dans ces deux catégories, cumulent 2875 occurrences soit $49 \%$ du total. Viennent ensuite les inscrits provisoires avec 673 cas. Les novices (752) et les mousses (195), ensemble, constituent (947) $16 \%$ des cas. 
Tableau 2: Statuts des hommes inscrits au matricule et victimes d'accidents maritimes, venant pêcher à Terre-Neuve

\begin{tabular}{|c|c|}
\hline Catégories d'inscriptions & Hommes \\
\hline Provisoires & 673 \\
\hline Marins & 418 \\
\hline Matelots le classe & 198 \\
\hline Matelots 2e classe & 543 \\
\hline Matelots 3es classe & 1843 \\
\hline Matelots (sans mention de classe) & 1032 \\
\hline Novices & 752 \\
Mousses & 195 \\
\hline Hors classification & 202 \\
\hline
\end{tabular}

Tableau 3: Fonctions exercées par les hommes inscrits au matricule et victimes $d^{\prime}$ 'accidents maritimes, une fois sur les lieux de pêche ${ }^{93}$

\begin{tabular}{|c|c|}
\hline Catégories & Hommes \\
\hline Avant de doris & $633(32 \%)$ \\
\hline Patrons de doris & $642(33 \%)$ \\
\hline Maîtres ${ }^{*}$ & 240 \\
\hline Seconds & 108 \\
\hline$* *$ Patrons & 89 \\
\hline Capitaines & 65 \\
\hline Saleurs & 60 \\
Pêcheurs & 50 \\
Graviers & 46 \\
\hline
\end{tabular}

Quant aux catégories de fonctions des hommes une fois en pêche, elles se chiffrent à neuf pour un total de 1933 cas. Les avants de doris (633) regroupent $32 \%$ des cas, juste après les patrons de doris (642) avec 33\%. Il n'y a rien de bien surprenant à ces résultats à peu près égaux, puisque chaque doris est monté par un patron et un avant. Il y a donc une forte probabilité qu'ils soient impliqués ensemble dans la même embarcation lors d'un accident maritime. Tel que mentionné en note 93, la catégorie des maîtres (240) englobe une grande diversité de fonctions. Viennent ensuite des tâches typiquement associées aux pêches soit celles des graviers (46), des pêcheurs (50) et des saleurs (60). 
Le sort voudra donc qu'un nombre non négligeable de ces centaines de marins et pêcheurs traversant l'Atlantique, connaîtra un sort tragique. Chez ceux qui disparaissent, les cadavres sont parfois retrouvés ou encore, on connaît les lieux précis de leur disparition. Autrement dit, un ou des témoins confirment la noyade. Ici et plus loin dans le texte, j'ai recours à un échantillon d'hommes dont les noms de famille apparaissent sous les lettres $\mathrm{A}$ à $\mathrm{F}$ inclusivement. Il est utile de préciser que le détail des informations pour chaque homme perdu en mer peut varier entre minimale et bien fournie. Néanmoins, pour les principaux lieux de disparition confirmés ou de recouvrement des cadavres, cinq emplacements prédominent soit Saint-Pierre avec 54 (33\%), Langlade (16), Miquelon (9), l'île aux Chiens (9) et l'Île aux Vainqueurs (7). Le nombre total de cas observables se chiffre donc à 160, dont la grande majorité est associé à Saint-Pierre et ses 17 lieux tout autour de l'île. À noter que douze autres corps ont été retrouvés, mais le lieu de leur découverte n'est pas spécifié. Du côté de la côte de Terre-Neuve, on parle de 51 cas dont 10 à l'Île Rouge et 7 à Codroy.

\section{Disparaitre en famille}

Au-delà des pertes de vie individuelle, certains drames maritimes impliquent la disparition d'hommes apparentés; question déjà abordée pour le cas de Saint-Pierre-et-Miquelon. Quoiqu'un nombre substantiel d'exemples puisse être mis de l'avant, il n'en demeure pas moins qu'il n'est pas toujours possible d'établir l'exactitude des liens de parenté entre des hommes qui partagent le même nom de famille. Par exemple, citons le cas de quatre hommes du nom de Genu, tous de Cancale, tous étiquetés « disparus en mer » entre 1876 et 1898 . Deux d'entre eux connaissent ce sort fatal sur la goélette Ranger en septembre $1876^{94}$. Notons également les quatre Girardin dont trois sont nés à Saint-Pierre. Ces quatre hommes disparaissent entre 1833 et 1885 . Nous savons cependant que cette année-là, Jean-Baptiste et Joseph disparaissent le 27 août, sans que nous sachions comment, ni sur quelle embarcation. Joseph-Marie, lui, est sur le doris Nichette lorsqu'il se 
noie à la Pointe au Cheval de Miquelon. Et que dire des trois Huet, nés à Dinan et servant tous sur la goélette Isabelle Marie, présumée disparue en mer dans la grande tempête du 13 septembre 1900.

Après les Genu de Cancale et les Girardin de Saint-Pierre, penchonsnous sur les trois Colin de Binic soit François-Marie, Jean-Baptiste père et Jean-Baptiste fils, âgés respectivement de 36, 43 et 18 ans ${ }^{95}$. Les deux premiers sont patrons de doris et le troisième est un novice. On dira de leur navire qu'il est " présumé perdu corps et biens sans nouvelles depuis son départ à cette date ». Pour les deux autres exemples parmi les plus significatifs, on peut se référer aux frères Alphonse Marie et Frédéric Aimé Fiaut ${ }^{96}$ de Granville ou encore à Jean et Pierre Dubourg de Yquelon ${ }^{97}$. Les premiers disparaissent en 1886 et les deuxièmes en 1851. Finalement, Charles Marie Dairon, matelot de $3^{\mathrm{e}}$ classe sur la goélette Marie-Françoise, perd la vie dans la rade de Saint-Pierre en compagnie de « 5 autres hommes » dont Pierre Fossard ${ }^{98}$.

On peut imaginer la détresse envahissante des parents vivant dans l'attente du retour de membres de leurs familles, à la fin d'une campagne sur les Grands Bancs de Terre-Neuve ou à Saint-Pierre-et-Miquelon. Lorsque l'inévitable se présente, la nouvelle se répand rapidement et la douleur se veut encore plus vive si le ou les corps n'ont pas été retrouvés. Une certaine consolation est envisageable si l'on apprend que les disparus ont bénéficiés d'un enterrement religieux dans l'archipel. Mais l'angoisse de l'attente se manifeste tout autant lorsque la famille demeure dans l'incertitude. Autrement dit, a-t-on oui ou non été en mesure de confirmer l'identité du ou des disparus? Question que j'aborde dans la section suivante.

\section{Identité incertaine?}

Dans un contexte de commerce maritime et de pêche s'étendant sur un aussi grand territoire et impliquant un aussi grand nombre d'hommes, faut-il sétonner qu'il surgisse parfois des doutes sur l'identité de certaines victimes d'accidents maritimes? Ainsi, en 1842, Jean Dilly, 46 ans, de Lannion, décède à bord de la goélette Virginie. Mais l'on men- 
tionne qu'il est « Dit aussi embarqué sur canot no 20 ». Serait-il plutôt décédé à l'Ile-aux-Morts à Terre-Neuve ${ }^{99}$ ? Une dizaine d'années plus tard, Jean Charles Farvaque disparait alors qu'il est en service sur la goélette Eugénie. On estime qu'il serait né vers 1821, donc âgé de 35 ans et marié. Toutefois, une certaine hésitation se dessine lorsque l'on mentionne que son prénom serait « probablement » Charles Jean, né à Saint-Pierre le 21 janvier 1819. Il aurait donc plutôt 37 ans ${ }^{100}$ ? C'est le même genre d'incertitude qui plane autour de l'identité et du destin de Jean Gille Brocqueville en 1892. Né à Cancale, il est sur la goélette Jacques Cartier au moment où il disparaît. Les autorités pensent cependant que c'est « sans doute le même homonyme qui avait disparu au début de 1886 » et quon pensait embarqué sur une goélette américaine ${ }^{101}$. Pour Louis Léopold David, 30 ans, de Saint-Pierre en Fort, on dira qu'il est " peut-être décédé à bord », le 16 avril $1857^{102}$.

Cette incertitude quant à l'identification des victimes d'accidents maritimes, s'exprime aussi au moment de statuer sur l'identité des cadavres. Ainsi, Yves Marie Bodiou, de Lannon, est d'abord déclaré " cadavre inconnu » au moment où il a été trouvé le 26 août 1896 soit 20 jours après son constat de disparition à Barachois ${ }^{103}$. Quant à Emmanuel Auguste Colin, de Saint-Malo, on signale qu'il est rapporté mort au Barachois le 12 juin 1900. Son corps est retrouvé environ un mois plus tard, quoique l'on mentionne que le « cadavre retrouvé le 14 juillet est supposé être le sien ${ }^{104}$. C'est à peu près le même constat pour Guyot le 8 juillet 1891, lorsqu'on retrouve un cadavre « inconnu, supposé être Guyot». On avait signalé sa disparition environ trois mois auparavant, lui accolant le statut d' " absent illégalement » au Barachois. Son décès est finalement inscrit à l'île aux Chiens ${ }^{105}$. Un autre homme à l'identité incertaine est Alfred Hamon, puisqu'un autre individu porte ce nom au registre des matricules. Ils sont tous deux nés à Pélan le Petit en juin 1890, l'un le 27 et l'autre le 21. L'un disparait en 1907 et l'autre en 1912. Mais s'agit-il bien du même homme ${ }^{106}$ ? Finalement, pour Alcide François Allory, d'abord déclaré disparu, on se ravise en disant qu'il « n'est pas perdu, suivant nouvelles officieuses ${ }^{107}$. 
Bien entendu, certains noyés parcourent d'importantes distances entre le moment de leur disparition et le recouvrement de leur corps. C'est ce qui se produit avec Jean-Pierre Boulin en 1891. Originaire de Saint-Malo, il monte le wary ${ }^{108}$ J.B. et le 2 septembre il est « présumé noyé dans les parages de l'île aux Chiens ». Mais son corps est plutôt retrouvé sur la côte de Terre-Neuve ${ }^{109}$.

Pour terminer cette section, penchons-nous sur les pertes de vie résultant des abordages avec d'autres navires, souvent d'une autre nationalité. Au milieu des années 1880, Paul Durel, de Granville, est sur le trois-mâts Michel Émile lorsqu'il disparait victime d'un « abordage par un anglais ». Sur le même navire et lors du même événement, meurt aussi Étienne Auguste Esnout de Saint-Rémy des Landes ${ }^{110}$. En juillet 1905, Auguste Desjardins, de Saint Valéry en Caux, sert à bord du brig Roland de Granville lorsqu'il décède parce qu'il " a sauté à bord du navire abordeur ", sans que l'on précise lequel ${ }^{111}$. En avril 1884, cette fois, un abordage survient entre la goélette Paquebot No 2 de Saint Servan et un navire norvégien. La collision cause au moins six morts du côté français ${ }^{112}$. Deux incidents sont également dignes de mention au début du $20^{\mathrm{e}}$ siècle. D'abord en 1905 lorsque Pierre Gervais, sur le Para, décide de sauter à bord d'un navire abordeur ${ }^{113}$. Finalement, en 1928, Marcel Augustin Donne, disparait lorsque " abordé par le chalutier Cdt Émaille ». Il exerce alors à titre de patron de doris ${ }^{114}$.

\section{Pas seulement des noyades}

Il est important de noter que certaines mortalités en contexte maritime ne sont pas attribuables à des noyades. En effet, tous ne connaissent pas une fin aussi typiquement maritime que Victor Jean Marie Bequet, trouvé mort «sur la bouée de ses lignes » en mai $1882^{115}$. N'empêche que certaines causes de décès demeurent violentes. D'abord, Pierre Baudouard, né vers 1802 (l'année précise n'est pas connue ${ }^{116}$ ), est abattu par un patrouilleur anglais dans sa pirogue № 309. Le même sort est réservé à Pierre Cosentin Delepine, de Cherrueix, « abattu d'un coup de fusil après avoir mis le feu à bord dans un accès de folie ». 
Il est alors avant de doris à bord du trois-mâts Valleda de Granville en $1893^{117}$. Le mécanicien Suédois Gustave Carlsson meurt de la même manière, en raison d'une malheureuse confusion survenue en 1928. Sa mort découle de l'action d'un gendarme de l'Archipel qui l'a « abattu en l'ayant confondu avec un contrebandier ${ }^{118}$. Travaillant à bord du chalutier Caspiran au moment du drame, il décède à l'hôpital de SaintPierre.

Quant à Pierre François Coepel, marin de Granville, il meurt à terre en janvier 1844. Mais on semble ignorer la cause du décès lorsqu'il est trouvé « mort entre les maisons, rue Jacques Cartier » à SaintPierre $^{119}$. Un autre dont la vie prend fin dans la rue Jacques Cartier est James Cusick de St. Lawrence (Terre-Neuve) en novembre $1897^{120}$. Toujours à Saint-Pierre, cette fois « au calvaire dans le haut de la ville » est « retrouvé mort » Jean-Baptiste Ernouf, de Blainville ${ }^{121}$. Finalement, seul événement de ce genre enregistré dans le cadre de cette recherche, le mécanicien Jean-Baptiste Autier décède à bord en mai 1881 en raison d'une « descente opérée au moyen d'un scaphandre ${ }^{122}$.

L'approche d'échantillonnage appliqué dans des sections précédentes, s'avère tout aussi pertinent dans le cas d'hivernants décédant dans l'archipel mais chez l'habitant, ou au service d'une entreprise métropolitaine ayant des infrastructures dans la colonie. C'est donc toujours en fonction du même échantillon que j’ai relevé 49 décès survenus chez 42 habitants et 12 dans trois entreprises de pêche métropolitaine basée à Saint-Pierre. Chez les habitants, la veuve Audouze, rue Beaussant à Saint-Pierre, est témoin de cinq décès d'hivernants sous son toit. Les veuves Leflem (rue Borda) et Julien Briand (rue Ange Gautier) signalent chacun deux décès. C'est à l'habitation de la Compagnie Générale Maritime que le nombre de décès est le plus élevé avec neuf.

Ce passage démontre que la notion de risque maritime va au-delà de la vie à bord. Les marins à terre peuvent eux aussi se voir exposés à certains dangers découlant de bagarres, d'excès de boisson ou d'exposition au froid. Au risque d'avoir recours à des clichés historiographiques, souvenons-nous de ces nombreux récits de matelots débarquant dans 
les ports pour fréquenter les tavernes et les prostituées, et risquant de dilapider leur paye et faire des rencontres pouvant leur être fatales ${ }^{123}$. On doit donc en conclure que le risque maritime peut inclure de mourir les deux pieds sur terre et par beau temps!

Avant de passer à la question des victimes recueillies, il n'est pas sans intérêt de mentionner qu'un certain nombre d'hommes figurant au registre à titre de victimes d'accidents maritimes, perdent la vie ailleurs que dans le golfe du Saint-Laurent ou dans les environs de Terre-Neuve. Qu'il soit permis de mentionner trois cas représentatifs soit Auguste Marie Cressant ${ }^{124}$ en Islande, Auguste Célestin Bertrand $^{125}$ à Cadix en Espagne et Jean-Baptiste Bouder ${ }^{126}$ à l'hôpital d'Halifax en Nouvelle-Écosse.

\section{Les disparus recueillis}

Tel que mentionné plus haut, rappelons que les victimes d'accidents maritimes ne sont pas toujours disparues définitivement. Du moins c'est le cas pour 132 hommes recueillis, conformément à un échantillon incluant les noms de famille sous les lettres $\mathrm{A}$ à $\mathrm{F}$ inclusivement. Il est bon de rappeler que l'échantillon a été dressé à partir du registre des marins disparus et décédés. Quoiqu'il ne soit pas toujours possible de statuer sur ce qui survient après un sauvetage, je suis néanmoins en mesure de déterminer les trois destinations prédominantes de ces rescapés : retour à bord (20), retour à Saint-Pierre-et-Miquelon (70), ou encore en France (10). Quoique ces scénarios aient souvent tendance à se recouper, il demeure difficile de déterminer une séquence fiable. Autrement dit, un homme peut être rescapé en mer par un navire allant directement en France ou vers Saint-Pierre-et-Miquelon. Dans ce dernier cas, le marin rescapé peut opter pour se réembarquer à même l'archipel, ou sur un navire en partance pour la France. Bref, chacun tente du mieux qu'il peut de contrôler sa destinée.

Allons maintenant à la rencontre de ces disparus recueillis. D'abord, Sévère François Auguste David, de Plouër, est porté disparu en octobre 1894 alors qu'il monte la goélette L.H.B. de Saint-Malo.Il est cependant 
recueilli par un vapeur allant en Irlande et « rapatrié dans ses foyers », en France. Mais deux ans plus tard, en juillet 1896, il disparait à nouveau alors qu'il est employé sur la même goélette ${ }^{127}$. Son histoire ressemble beaucoup à celle d'Henri François Desbois, recueilli par le vapeur allemand Gut Heil et ramené à Hambourg. Il avait disparu le 29 août 1894, alors qu'il travaillait à bord de la goélette Marie Louise ${ }^{128}$. La fatalité frappe aussi Louis Jules Désiré-Delalande, de Saint-Georges la Rivière, qui disparait une première fois en juillet 1888 alors qu'il monte le brig Albertina. Chanceux à la manière de S.F.A. David, il est recueilli et ramené à Saint-Pierre. Mais la malchance le rattrape neuf ans plus tard, en septembre 1897, lorsqu'il disparaît à nouveau. Cette fois, il montait la goélette Saint-Pierrais. On dira d'elle qu'après son départ, " elle n'a pas reparu ». Contrairement à d'autres cas abordés dans ce texte, le décès de Désiré-Delalande est « déclaré constant » le 22 janvier 1902 à Saint-Pierre ${ }^{129}$. Ce qui signifie qu'il est définitivement confirmé, contrairement à d'autres cas évoqués plus hauts où les autorités semblent hésitantes à fermer un dossier de marin disparu. Rappelons que les rumeurs suivant un naufrage ou une disparition, peuvent parfois se prolonger sur d'assez longues périodes.

Un autre marin disparaissant à deux reprises est Zacharie François Guillet, premièrement en août 1881 et ensuite en avril 1884. D'abord porté disparu alors qu'il est à bord de la goélette Georges, il rentre à Saint-Pierre le 15 septembre. La deuxième fois, il monte la goélette Ernestine lorsqu'on assume qu'il est « sans doute parti en dérive ». Il est toutefois récupéré le $1^{\text {er }}$ mai par la goélette Namouna sur laquelle il décède. Porté disparu le 27 avril, l'on ignore ce qui était advenu de lui entre cette date et le $1^{\text {er }}$ mai $^{130}$.

Le cas de François Michel Auffray est des plus intéressant. Il est d'abord porté disparu le 7 mai 1890 à titre d'avant de doris sur la goélette Joséphine de Saint-Malo. On pense toutefois qu'il fut recueilli le même jour mais avec un brin d'incertitude. Cela s'explique puisqu'un autre homme du même nom, né le même jour et au même endroit, monte la goélette Anita, elle-aussi de Saint-Malo. Ce dernier disparait en août 1908, avant dêtre recueilli puis amené à Saint-Pierre 
le 3 septembre. Mais son périple ne s'arrête pas là puisqu'on le retrouve ensuite sur le Casimir Périer, toujours de Saint-Malo. Cette fois, il disparait en mer le 30 juin 1912 et, encore une fois, est recueilli le même jour et déposé à Liverpool (Angleterre) avant d'être rapatrié en France. La seule différence que l'on puisse établir entre ces deux hommes du même nom, est que le premier est classé à Saint-Pierre et le deuxième à Binic ${ }^{131}$. Finalement, à la fin de la période à l'étude, en 1923, Édouard Joseph Dubois disparait d'abord sur le trois-mâts $M i$ chel Boisselier, de Saint-Malo. Recueilli le même jour par le navire hôpital, il rentre « à bord ». Ce patron de doris disparait à nouveau en juin 1929, alors qu'il est rattaché au trois-mâts Santa Maria, aussi de Saint-Malo ${ }^{132}$.

Il y a ici lieu de revenir sur l'ensemble des destinées variées attendant les victimes d'accidents maritimes lors des campagnes de pêche. À l'exception des marins formellement disparus, dont les corps peuvent ou non être récupérés et dûment identifiés, il y en a qui, une fois recueillis, tentent de réembarquer. À l'occasion, la malchance les poursuit et ils peuvent subir un ou même deux autres accidents et même être victime de la fatalité. Les pires tragédies impliquent la disparition simultanée de membres d'une même famille. Mais n'oublions pas que partir en campagne de pêche ne signifie pas forcément que l'on ne puisse pas perdre la vie autrement que par la noyade. On peut tout autant succomber à la maladie, aux accidents tels les abordages ou mourir les deux pieds sur terre quelque part dans l'archipel. Par exemple, au service d'un habitant ou d'une compagnie métropolitaine ${ }^{133}$.

\section{Conclusion}

Cette recherche porte sur le phénomène des accidents maritimes survenus dans les eaux du golfe du Saint-Laurent, de Terre-Neuve et de Saint-Pierre-et-Miquelon entre 1817 et 1948. Les données servant à mesurer ce phénomène provenaient d'une source fondamentale soit le registre des marins disparus et décédés. Il a ainsi été possible d'identifier 6448 déclarations d'incidents maritimes affectant un marin, un 
officier ou un travailleur des pêches ou gens de mer, dont 248 se rapportent exclusivement à l'Archipel entre 1816 et 1910. À partir de ces déclarations, il semblerait que 4249 cas, soit $65 \%$, impliquent de fortes chances qu'il y ait eu disparition définitive en mer. C'est surtout le cas pour la période 1873-1900, alors que l'on relève 983 « disparitions en mer » soit 23\% du total, dont 250 en 1873.

Là où il est possible de confirmer mort d'homme c'est du côté des décès à bord des navires et dans les hôpitaux de Saint-Pierre-etMiquelon soit 2199 ou 34\% du total des déclarations d'accidents maritimes. La période allant de 1893 à 1903 est particulièrement funeste pour les décès à l'hôpital et les années 1900 à 1903 le sont tout autant pour les morts à bord des navires.

En ce qui a trait aux aspects descriptifs des accidents maritimes, les entrées du registre se contentent de catégoriser les circonstances de la disparition. Les termes utilisés permettent de constater si la disparition semble définitive ou incertaine. Parmi d'autres constats, mentionnons que parmi les 5419 déclarations de ports d'embarquement, 26\% indiquent Dinan, suivi de Cancale et de Saint-Malo. On est également en mesure de compiler des données à partir de neuf catégories de matricules chez les 5856 hommes classés. Il a aussi été possible de confirmer les sauvetages d'au moins 132 hommes chez les disparus. Rappelons que certains d'entre eux disparaissent en compagnie d'un parent, certaines notices de disparitions sont incertaines quant à l'identité des victimes et quelques hommes meurent autrement que par noyade.

Le survol historiographique de cette recherche permet de mieux cerner certaines composantes de l'univers maritime des pêcheurs. S’il demeure fort difficile d'établir si leur statut socio-économique s'améliore, certaines innovations technologiques marquent la période à l'étude. Les traversées deviennent moins longues et plus sécuritaires en raison de l'avènement progressif des navires à vapeur et d'infrastructures de santé. C'est ainsi que de meilleurs soins peuvent être prodigués à bord, sur un navire hôpital ou dans un hôpital de port, entre autres à Saint-Pierre. Mais en dépit de cela, les accidents maritimes 
demeurent fréquents et assujettis aux tempêtes, aux accidents et à la maladie à bord, aux abordages, ou même dans l'archipel.

La suite logique de cette recherche consistera à s'intéresser davantage au traitement journalistique des accidents maritimes dans l'Archipel et aux activités de commémoration de ces tristes événements.

\section{Notes}

1 «Démographie de l'île Miquelon 1816-1850», Newfoundland and Labrador Studies, Vol. 31, n 2, 2016, p. 286-315. «Événements démographiques chez une population coloniale française dans l'Atlantique : l'T̂le Saint-Pierre (1763-1791) (1816-1822) », Port Acadie, № 29, printemps 2016, p. 7-37. « Les dossiers personnels des réfugiés de l'île Royale et de Saint-Pierre-et-Miquelon au Ministère de la Marine et des Colonies, 1763-1815 », Revue de la culture matérielle, 86 (Automne 2017), p. 48-64. « Portrait démographique d'une colonie française d'Amérique : Saint-Pierre-et-Miquelon, 1763-1828 », Les Cahiers. Société historique acadienne, Vol. 49, n 1, mars 2018, p. 5-27 «Événements démographiques à l'île de Miquelon, 1762-1791 », Les Cahiers. Société historique acadienne, vol. 49, n 4, décembre 2018, p. 157-164.

2 Bernard Michon, «Les marins du Havre ", Histoire urbaine, 2017/2, n 49, p. 180.

3 Gérard LeBouëdec estime que le système des classes comporte des conséquences allant jusqu’à « hypothéquer l'avenir de certaines paroisses ». Le quotidien de ces hommes et de leurs familles serait hanté par « la mort, la détresse, la destruction des outils de travail ». "Le temps des crises dans les sociétés littorales (XVIe-XIXe siècle) ", Annales de Bretagne et des Pays de l'Ouest, 117-3/2010, p. 3.

4 "The Environmental Historiography of the Maritime Peninsula ", Acadiensis, vol. 45, n 1, Winter / Spring 2016, p. 163-177.

5 Robert Viau, «Le comte et les pêcheurs ; voyage à Terre-Neuve d'Arthur Gobineau ", Studies in Canadian Literature / Études en littérature canadienne, vol. 41, n 2, 2016, p. 6.

6 Jean-Michel Auffray, « De la terre à la mer, les frères Barbedienne ", Annales de Bretagne et des Pays de l'Ouest, 115-4/2008. Varia, p. 82-83.

7 C'est du moins l'opinion de LeBouëdec, qui met de l'avant la 
«pertinence du concept de pluriactivité des communautés littorales ». Il pense qu'il faille remettre en question « l'idée d'une identité maritime pure et dure ». Ces hommes cherchent surtout « un supplément de revenu pour la famille ». Bref, on parle plutôt de «nécessité que de vocation ». «Les petits ports de la façade atlantique (Du XVIe au XIXe siècle) », Revista da Faculdade de Letras, Historia, Porto, III, Série, vol. 9, 2008, p.11-12, 21.

8 Effectivement, des milliers d'hommes sont alors « encadrés par le système des classes » qui en font des « réservistes pour la flotte de guerre ». Toutefois, ce système les rend éligibles à certains avantages dont la « caisse des invalides », des soins médicaux « gratuits » et une petite pension. Thierry Sauzeau, « Les mousses ou garçons et la souffrance sociale (XVIIe-XIXe siècles) », dans Frédéric Chauvaud, Histoire de la souffrance sociale, Rennes, Presses universitaires de Rennes, p. 2. Voir en ligne http://books.openedition.org/pur/6696?lang=fr. (p.1-17). Consulté le 24 janvier 2018.

9 C'est ainsi que chaque embarquement permet aux gens de mer, en pêche, en commerce ou sur la Royale, de cumuler du « savoir-faire » et de « l'apprentissage ». N'empêche que les aptitudes individuelles tels « l'endurance et la force des bras » demeurent essentiels. Sauzeau, « Les mousses ou garçons... », op.cit., p.2.

10 Avec le temps, ces hommes peuvent devenir graviers, pêcheurs, décolleurs, habilleurs ou saleurs. Auffray, « De la terre à la mer... », op.cit., p. 86, 91. Claire Boer parle plutôt de « catégories de navigants » pour désigner les « maitres d'équipage, matelots qualifiés, charpentiers, calfats, voiliers ». « Les travailleurs de la mer : désertions et débarquements des marins provençaux au XVIIIe siècle », Tracés. Revue de sciences humaines, 32/2017. URL : http://traces.revues.

org/6826;DOI :10.4000/traces.6821, p.3. Consulté le 6 octobre 2017. Certains de ces métiers sont pratiqués dans les chantiers navals par des hommes qui, dans certains cas, accompagnent les navires de la grande pêche recherchant leur expertise pour l'entretien des navires. Voir Laurent Pavlidis, « Petits ports et chantiers navals traditionnels en Provence au XIXe siècle », Revue méditerranéenne, 35/2010, p. 45-59.

11 J.M. Auffray, « De la terre à la mer...», op.cit., p. 90, note 29.

12 Thierry Sauzeau, « La pluriactivité des marins du long cours au 
XVIIIe siècle ", Annales de Bretagne et des Pays de l'Ouest, 120-2/2013, p. 66. http://abpo.revues.org/2618; DOI : 10.4000labpo.2618. Consulté le 2 janvier 2017.

13 Alain Cabantous, «Le corps introuvable. Mort et culture maritime (XVIe-XIXe siècles) », Histoire, économie et société, vol. 9, n 3, 1990, p. 323.

14 Cabantous, «Les petits ports...», op.cit., p. 22.

15 Sauzeau, «Les mousses... », op.cit., p. 2. Selon cet auteur, à l'époque de la marine à voile, les « décès attestés représentent $15 \%$ des ruptures de carrière des mousses ", p. 10. D'autres matelots choisissent plutôt la désertion. Ainsi, à la fin du $19^{\text {e }}$ siècle, $3 \%$ des matelots de navires finlandais penchent pour cette option et $20 \%$ des matelots de navires canadiens font de même. Jari Ojala, Jaakko Pehkonen, Jari Eloranta, « Desertions in Nineteenth-Century Shipping: modeling quit behavior ", European Review of Economic History, vol. 17, Issue I, 1 février 2013, p. 122.

16 Nathalie Meyer-Sablé, Familles de marins-pêcheurs et évolution des pêches. Littoral morbihannais 1830-1920, Paris, L'Harmattan, 2005, p. 12.

1717 Guy Lemarchand, «La mer, les Français et les Normands depuis le Moyen Âge ». Alain Cabantous, André Lespagnol, Françoise Peron (dir.), Les Français, la terre et la mer XIIIe-XXe siècles. Dans Annales de Normandie, $55^{\mathrm{e}}$ année, $\mathrm{n}^{\circ}$ 5, 2005, p. 472-473.

18 Rodrigo de Oliveira Torres, «Handling the ship: rights and duties of masters, mates, seamen and owners of ships in nineteenth-century merchant marine ", International Journal of Maritime History, Vol. 26, no 3, 2014, p. 587-590.

19 Philip F. Reid, « Something Ventured: Dangers and risk mitigation for the ordinary British merchant ship, 1600-1800», The Journal of Transport History, vol. 38, n 2, 2017, p. 89.

20 Michon, «Les marins du Havre. », op.cit., p. 3. Selon Jean-Christophe Fichou, ce serait les villes abritant des conserveries de poisson qui, durant le $19^{\mathrm{e}}$ siècle, figurent parmi les « plus durement touchées par les épidémies de choléra ». "Les cités sardinières, portes ouvertes aux épidémies de choléra (1852-1914) ", Annales de Bretagne et des Pays de l'Ouest, 124-4/2017, Varia, p. 133.

21 Patrick Louvier, « Les maladies et les accidents dans la marine de 
guerre française en Méditerranée au XIXe siècle (1815-1914) », Corps, 2017/1, no 15 , p. 305.

22 Ibid.

23 Patrick Louvier, «Les maladies et les accidents dans la marine de guerre française en Méditerranée au XIXe siècle (1815-1914) », Corps, 2017/1, $\mathrm{n}^{\mathrm{o}} 15, \mathrm{p} .305$. Également, à la fin du $19^{\mathrm{e}}$ siècle, les cirés protègent contre l'humidité et il devient possible de laver et de sécher les vêtements des marins, du moins sur les plus grosses unités. Ibid., p. 307-308. À titre de référence canadienne sur l'habillement des pêcheurs, voir Sylvain Boudreau, «L'habillement du pêcheur gaspésien dans la première moitié du 19e siècle », Magasine Gaspésie, mars-juin 2016, p. 12.

24 C'est dès le milieu du $19^{\mathrm{e}}$ siècle que l'on rassemble les marins malades dans des lazarets pour contrer la contagion. Louvier, « Les maladies et les accidents... ", op.cit., p. 307-308.

25 Mark Harrison, "Merchant Seamen's health, 1860-1960-medecine, technology, shipowners and the state in Britain ", Journal of Maritime Research, 18 :2, 2016, p. 157-158.

26 C. Pearce, " Charity and Philantropy in a Coastal World: Scottish Fishing Communities and the Shipwrecked Fishermen and Mariners' Royal Benevolent Society, 1839-1848 », In : D. Worthington (eds.) The New Coastal History, Palgrave Macmillan, Cham. 2017, Springer International Publishing AG.

27 La Société des CEuvres de Mer. http://www-phil-ouest.com.

28 À la fin de cette campagne de pêche 1898, le bilan se chiffre à « 282 navires assistés, 57 malades hospitalisés et 14 naufragés recueillis ». Marine nationale, patrouilleur auxiliaire Sainte Jehanne, 1898, p. 2, 7.

29 Ibid.

30 Je remercie l'évaluateur no 2 de cet article pour ce rappel. Le lecteur peut se référer à l'article de Michael M. Barkham, « New Documents concerning the French Basque Pilot, Martin de Hoyarsabal, Author of the First Detailed Rutter for the New Found Land (1579) ", Newfoundland and Labrador Studies, vol. 19, Spring 2003, p. 103-131. Brad Loewen et Egaya Goya, « Le routier de Piarres Detcheverry, 1677. Un aperçu de la présence basque dans la baie des Chaleurs au XVIIe siècle ", Revue d'bistoire de l'Amérique française, vol. 68, no 1-2, été-automne 2014, p. 125-151. 
31 Précisons toutefois qu'à cet égard, des recherches s'intéressent à l'organisation de l'espace sur les navires. Les chercheurs tentent ainsi de reconstituer les dynamiques sociales de la société du bord. Le navire serait même un lieu d'interaction sociale, à l'intérieur du concept de paysage culturel maritime. Michael Joseph Moloney, « Re-imagining Shipboard Societies: A Spatial Approach to Analyzing Ships of the British Royal Navy during the 18th and 19th Centuries », University of Calgary, Thèse de doctorat en archéologie, 2015, p. 11, 28.

32 Bryn Tapper, «An Archaeological analysis of the distribution of French Fishing rooms on the Petit Nord, Newfoundland », St.John's, Newfoundland, Memorial University, thèse de maîtrise, 2014, p. 27.

33 Tapper, «An Archaeological... », op.cit., p. 49, 51.

34 Annika Korsgaard et Martin Gibbs, « Shipwrecks as Archaeological Signatures of a Maritime Industrial Frontier in the Solomon Island, 1788-1942 », International Journal of Archaeology, 2016, 20, p. 111.

35 J.M. Auffray, « De la terre à la mer... », op.cit., p. 83, 85-86.

36 James Candow, «An Overview of the Northwest Atlantic Fisheries, 1502-1904 », Northwest Atlantic Fisheries. The Sea in European History, 2006, p. 163-184. En ligne.

37 J.M. Auffray, « Entre grande pêche et commerce. Les reconversions des armements bretons sous le Second Empire ", Annales de Bretagne et des Pays de l'Ouest, 117-4/2010, p. 1.

38 J.M. Auffray, « De la terre à la mer... », op.cit., p. 96. Au sujet des tensions régnant sur la côte de Terre-Neuve durant la première moitié du $19^{\mathrm{e}}$ siècle, voir Nicolas Landry, «Tensions, diplomatie et accommodements dans un espace partagé entre Saint-Pierre-et-Miquelon et Terre-Neuve, 1816-1842 ", The Northern Mariner/le marin du Nord, XXVII, no 2, avril 2017, p. 143-162.

39 Philippe Fournet, «La grande pêche morutière française ». In Travaux de l'Institut Géographique de Reims, no 33-34, 1978. Aspects de la pêche industrielle, p. 42.

40 Ce serait en 1871 qu'arrivent les lignes de fonds pouvant atteindre 1646 mètres. Candow, op.cit., p. 177.

41 À noter qu'à partir de la Révolution, la pêche se fait au moyen de grandes chaloupes œuvrant en mer, à proximité d'un navire et utilisant des lignes de fonds. Les doris, eux, apparaissent plutôt après 1870, sont 
plus légers que les chaloupes et montés par deux hommes. La pêche de chaque doris fait l'objet d'un partage à raison de « $5 / 9$ pour le patron et 4/9 pour l'avant de doris ». Charles de La Morandière, « Le port de Granville des origines à nos jours. L'avenir », Études normandes, livraison $15, \mathrm{n}^{\circ}$ 50, $2^{\mathrm{e}}$ trimestre 1955, p. 259. À celui-là s'ajoute bientôt un doris de pêche côtière à partir de l'Archipel, dont quelques-uns sont de plus grande taille et munis d'une voile. Aliette Geistdoerfer, «Des lions, des mouettes et des fauvettes. Étude des patronymes des doris de Saint-Pierre. Des rapports entre les pêcheurs et la mer », Cabier des Annales de Normandie, no 26, 1995. Mélanges René Lepelley, p. 194.

42 Philippe Fournet, « La grande pêche morutière française ». op.cit., p. 42.

43 Tapper, «An Archaeological Analysis... », op.cit., p. 14.

44 Alan Ruffman, «The Multidisciplinary Rediscovery and Tracking of The Great Newfoundland and Saint-Pierre-et-Miquelon Hurricane of September 1775 », The Northern Mariner/le Marin du Nord, VI, n 3, July 1996, p. 19. À la même époque, en 1859, Gobineau rapporte le cas d'un patron de bateau pilote de Saint-Pierre ayant, à lui-seul, à 60 ans, déjà perdu quatre fils à la noyade. Ronald Rompkey (ed.), Les Français à Terre-Neuve. Un lieu mythic, une culture fantôme. Bordeaux, Presses universitaires de Bordeaux, 2009, p. 199.

45 Christian Bousquet-Labouérie, compte-rendu du livre de Xavier Daumalin, Daniel Faget et Olivier Raveaux (dir.), La mer en partage: sociétés littorales et économies maritimes XVIe-XXe siècle, Lectures (En ligne), Les comptes-rendus, 2016, mis en ligne le 1er septembre 2016. Consulté le 23 février 2018. http://journals.openedition.org/lectures/21266. P.2.

46 Annika Korsgaard et Martin Gibbs, « Shipwrecks as Archaeological Signatures of a Maritme ». op.cit., p. 112.

$47 \quad$ Ibid., p. 117.

48 Ibid., p. 119-120. Au moins 64\% des naufrages observés dans leur étude résultent de facteurs environnementaux, surtout des échouages et des tempêtes. À cela s'ajoutent des facteurs humains tels que les pauvres compétences de navigation, des outils de navigation peu précis et les fuites d'eau. Pages 120-121.

49 Brad Duncan et Martin Gibbs, Please God send me a wreck. Response to shipwreck in a 19th century Australian Community, New York, Springer- 
Verlag, 2015, p. 18-21. Cet ouvrage examine 90 naufrages survenus sur la côte de Queens Cliff en Australie.

50 Marc Pabois, « L'archipel de Saint-Pierre-et-Miquelon », In Situ. Revue des patrimoines, 3/2003, p. 1 et 4 . Aux îles, les principales « écoueries » se trouvent à Saint-Pierre (l'Anse à Rodrigue, l'Anse à Savoyard, l'Anse à l'Allumette, l'Anse à Philibert), à l'île aux Marins, à Langlade et à Miquelon (Petit Barachois et Pointe du Cheval). Jacques Ancellin, «La pêche aux îles. Rapport de mission, mai à juillet 1951 », Revue des travaux de l'Institut des pêches maritimes, 19 (1), 1955, p. 24.

51 Ancellin, « La pêche aux îles... », op.cit., p. 33.

52 André-Louis Sanguin, «II. Un milieu insulaire et nordique », Norois, vol. $110, \mathrm{n}^{\circ} 1,1981$, p. 155.

53 L'abbé Haristoy, Les paroisses du Pays Basque pendant la période révolutionnaire. Tome I, Imprimerie Vignancour, 1895, p. 428.

54 Émile de Curton, Saint-Pierre-et-Miquelon, Grand Colombier, Imprimerie nord-africaine Drouillet, Alger, 1944, p.7.

55 André-Louis Sanguin, «Un archipel balloté entre deux puissances coloniales ", Norois, vol. 110, n 1, 1981, p. 139.

56 «L'histoire de Saint-Pierre-et-Miquelon », https://www.futura-sciences. com.

57 Fr.wikipedia.org/wiki/Saint-Pierre-et-Miquelon.

58 Jean-Pierre Le Gléau, « Le recensement de la population à SaintPierre-et-Miquelon », INSEE (Institut National de la statistique et des études), nº 73-Août 2000, 3 pages.

59 Marc Dérible, « Saint-Pierre-et-Miquelon : origines et originalités », Cabiers. Société historique acadienne, vol. 32, n 4, 2001, p. 218. En 1887, il y aurait 187 navires métropolitains mouillés dans le port de SaintPierre. Sanguin, «Un archipel balloté... », op.cit., p. 141.

60 À titre d'exemples, mentionnons La Compagnie Générale Maritime Théodule et Lecampion qui, en 1861, devient la Compagnie Générale Transatlantique. La Morandière, op.cit.., p. 258. Également, la production d'huile de foie de morue se commercialise dans l'Archipel à compter de 1850. En 1905 est formée la société La Morue Française et en 1918, la Société Morue Française et Sécherie de Fécamp. Charles Guyotjeannin et Joseph Lehuenen, « Les îles Saint-Pierre-etMiquelon productrices d'huile de foie de morue », Revue d'bistoire de 
la pharmacie, $73^{\mathrm{e}}$ année, n 264,1985 , p. 53-55.

61 Émile de Curton, Saint-Pierre-et-Miquelon, op.cit., p. 40.

62 Sanguin, «Un archipel balloté... », op.cit., p. 139-141. Au début du $19^{\mathrm{e}}$ siècle, ces goélettes sont munies de « deux mâts inclinés en arrière, la voilure comporte des focs, une grande voile et une misaine de forme trapézoïde enverguées à une corne et surmontées de huniers ». De Curton, Saint-Pierre, op.cit., p. 35.

63 Ronald Rompkey (ed.), Les Français à Terre-Neuve, op.cit. p. 174.

64 De Curton, Saint-Pierre, op.cit., p. 38.

65 Ancelin, « La pêche aux îles Saint-Pierre-et-Miquelon », op.cit., p. 218.

66 Sanguin, «II. Un milieu insulaire et nordique...», op.cit., p. 158-161. De Curton va même jusqu'à écrire que «l'armement saint-pierrais de la grande pêche a pour ainsi dire disparu » rendu en 1920. Saint-Pierre, op.cit., p. 38.

67 Geistdoerfer parle de 465 doris en 1900, de 310 en 1920 et de seulement 176 en 1940. Op.cit., p. 193.

68 Ancelin, « La pêche aux îles Saint-Pierre-et-Miquelon », op.cit., p. 218.

69 Cette réflexion s'inspire de commentaires formulés par l'un des évaluateurs de ce texte.

70 Séguin, «II. Un milieu insulaire... », op.cit., p. 158, 161.

71 Ancelin, « La pêche aux îles... », op.cit., p. 218.

72 De Curton, Saint-Pierre-et-Miquelon, op.cit., p. 7.

73 Marins disparus et décédés. 26 octobre 1817. Jean-Édouard Poirier. Né en 1791, il meurt à 26 ans.

74 Marins disparus et décédés. 25 août 1873. Édouard-Antoine Enguehard. Goélette Jeanne Marie Joseph. 25 août 1873. Pierre Théophile Enguehard. Goélette Brunette.

75 Marins disparus et décédés. 13 septembre 1900. Eugène Joseph Frioult. Goélette Fiona. Le frère d'Eugène Joseph, soit Eugène Marie Auguste, 25 ans, meurt sept ans plus tard à bord de la goélette Madeleine. Marins disparus et décédés . 30 mars 1907. Goélette Madeleine. On pourrait aussi mentionner les frères Joseph et Jean- Baptiste Girardin, disparaissant tous deux le 27 août 1887.

76 Marins disparus et décédés. 13 septembre 1900. Xavier Yves et Joseph Victor Lafourcade. Goélette Ali Baba.

77 Marins disparus et décédés. 18 juin 1908. Gratien Jugan. Goélette Boer. 
78 Marins disparus et décédés. 1 mai 1891. Georges Bonnel.

79 Marins disparus et décédés. 30 avril 1884. George Himberton.

Goélette O. Luther. Il serait né à Burin vers 1851 .

80 Marins disparus et décédés. 28 novembre 1897. James Cusick. Mort à 38 ans.

81 Marins disparus et décédés. 1 avril 1856. Thimoté Martin. Goélette Reciprocity (Halifax).

82 Marins disparus et décédés. 4 octobre 1878. Dominique Dacosta.

83 Marins disparus et décédés. 22 février 1871. Pierre Jean-Baptiste Lelorieux.

84 Marins disparus et décédés. 19 juillet 1886. Jean Toben. Ce Lazaret est alors situé sur la « route Iphigénie ».

85 Marins disparus et décédés. 19 mai 1876. Edouard Turpin.

86 Arche-musee-et-archives.net/fr/71_presselocale.html.

87 «Les marins disparus et décédés ». http://www.arche-musee-etarchives.net. Site hébergé par le Musée de Saint-Pierre-et-Miquelon. Il s'agit d'un inventaire non exhaustif et régulièrement mis à jour. Les informations composant cet inventaire proviennent de « deux registres recensant les disparitions et les décès des inscrits maritimes, de la collection des procès-verbaux des disparitions et d'inventaires de coffres des marins, de l'état civil, des états annuels des mouvements des marins des quartiers métropolitains et des rôles d'équipages. L'inventaire est organisé par ordre alphabétique des noms de famille des marins, pêcheurs, officiers, etc. Pour chacun d'entre eux ou presque, on trouve « l'identité du marin, le quartier, le folio et le numéro d'inscription, le nom du navire, le port d'enregistrement, la date et la raison de la présence dans l'inventaire ».

88 Notons que les termes utilisés pour rapporter des accidents maritimes varient passablement. Citons-en quelques-uns : goélette supposée disparue dans le coup de vent à cette date, goélette partie à cette date et qui n'a plus reparu, goélette retrouvée chavirée, sans nouvelles depuis cette date, goélette perdue corps et biens, etc.

89 Ces chiffres sont ceux de l'hôpital de Saint-Pierre, excluant ceux du Lazaret de l'Île-aux-Vainqueurs.

90 Par exemple, à Cancale, la grande tempête du 22 septembre 1866 est qualifiée de « jour de colère sur les bancs » sans pour autant fournir de 
chiffres sur l'ampleur du désastre. On mentionne toutefois les noms de 16 bâtiments de types goélettes, bricks et brick-goélette. Cette même source mentionne que de 1826 à 1897, il serait survenu 11 naufrages de bâtiments durant les traversées de Cancale à Saint-Pierre, soit sur les bancs ou encore dans les environs de Terre-Neuve et de SaintPierre-et-Miquelon. Par exemple, en mars 1889, le trois-mâts le Quatre Frères « se perd corps et biens », faisant 115 victimes. « Naufrages et naufragés». Source: Les Cahiers de la vie à Cancale, AABUC. Dictionnaire des marins de Cancale. Association Parchemin. En ligne. Au sujet de cette même tempête, voir http://www.saintpierreetmiquelon.net/22091866-la-grande-tempete22/09/1866.

91 Sauzeau explique que si quelques « très jeunes garçons s'embarquent à 7 ou 8 ans, la majorité sont âgés entre 12 et 16 ans ». Il ajoute quen moyenne, « la carrière de mousse » pouvait osciller autour de « 5,5 ans » soit le temps requis pour effectuer « trois voyages ». Sauzeau, « Les mousses ou garçons et la souffrance... », op.cit., p. 5-17.

92 À ce sujet voir Fournet, op.cit., p. 42. Également, Abbé J.-M. Grossetête, La Grande Pêche à Terre-Neuve et d'Islande, Saint-Malo, Éditions l'Ancre de Marine, 1988.

93 À noter que certaines de ces grandes catégories cachent de nombreuses sous-catégories qu'il est important de ne pas ignorer. D'abord, les capitaines inclus dans ce tableau peuvent tout aussi bien exercer au long cours qu'au cabotage. Chez les patrons** (excluant ceux clairement identifiés comme étant aux commandes d'un doris), on inclut ceux en charge d'un wary, d'une pirogue, d'un canot, d'une goélette ou encore d'un sloop. La situation est encore plus complexe chez les maîtres* où l'on en a en cabotage, en pêche, de chaloupe, d'équipage et les maitres-voilier. Il y a aussi les contre-maitres, les quartiers-maitres, les premiers maîtres ou encore les maitres de manœuvres. Rappelons que le wary est une variante du doris mais qui disparait progressivement durant la première moitié du $20^{\mathrm{e}}$ siècle. Il est monté par deux pêcheurs, souvent apparentés, et peut contenir de 4000 à 5000 livres de morue. Geistdoerfer, op.cit., p. 197.

94 Marins disparus et décédés. Deux hommes de ce nom apparaissent sur la goélette Ranger en septembre 1876. Pour les Girardins. 13-9-1900. Les Huet de Dinan sont sur la goélette Isabelle Marie. 
95 Marins disparus et décédés. 14 avril 1896. Sloop P.F. № 42. Les Colin.

96 Marins disparus et décédés. 20 août 1886. Brig-goélette Julien Gabrielle de Granville. Alphonse Marie (27 ans) et Frédéric Aimé Fiaut (32 ans).

97 Marins disparus et décédés. 27 août 1851. Goélette Églantine. Jean et Pierre Dubourg.

98 Marins disparus et décédés. 16 septembre 1859. Goélette Marie-Françoise. Charles Marie Dairon et Pierre Jean Fossard. Certaines circonstances rendent difficile de confirmer la cause exacte du décès. Pensons à Victor Louis Durand, 30 ans, de Saint Cast. Il est quartier-maître en 1891 lorsque son corps est retrouvé dans la chambre du navire ramené dans le port de Saint-Pierre. Sa mort est-elle attribuable à un accident de travail, à la maladie ou encore a-t-il simplement été repêché après une noyade? Marins disparus et décédés. 3 août 1891. Goélette Auguste Léontine. Victor Louis Durand.

99 Marins disparus et décédés. 25 avril 1842. Goélette Virginie. Jean Dilly.

100 Marins disparus et décédés. 15 décembre 1856. Goélette Eugénie. Jean Charles Farvaque.

101 Marins disparus et décédés. 1 décembre 1892. Goélette Jacques Cartier. Jean Gilles Brocqueville.

102 Marins disparus et décédés. 16 avril 1857. Louis Léopold David.

103 Marins disparus et décédés. 6 août 1896. Goélette Belle. Yves Marie Bodiou.

104 Marins disparus et décédés. 12 juin 1900. Goélette Aristide. Emmanuel Auguste Colin.

105 Marins disparus et décédés. 8 juillet 1891. Goélette J.B.C. Guyot.

106 Marins disparus et décédés. 1907 et 1912. Alfred Hamon.

107 Marins disparus et décédés. 18 juillet 1886. Brig Louis. Alcide François Allory.

108 Le wary est une petite embarcation à fond plat montée par deux hommes, le patron et l'avant, destinée à la petite pêche à Saint-Pierreet-Miquelon et à Terre-Neuve. Un décret du 17 septembre 1881 reconnaît formellement ce type d'embarcation. Voir Annuaire des îles Saint-Pierre-et-Miquelon, Saint-Pierre, Imprimerie du gouvernement, 1891, p. 29. Robert de Loture, Histoire de la Grande Pêche de TerreNeuve, Saint-Malo, l'ancre de Marine, 1994, p. 254. 
109 Marins disparus et décédés. 2 septembre 1891. Wary J.B. Jean-Pierre Boulin. Parmi de nombreux autres « présumé noyé » ou disparu peuvent s'ajouter Toussaint Georges Briot de Dinan en 1875 ou encore Joseph Coassin de Binic en 1890. Marins disparus et décédés. Août 1875. L'Élisabeth Joseph. Toussaint Georges Briot. 31 mai 1890. Joseph Coassin.

110 Marins disparus et décédés. 8 juin 1886. Trois-mâts Michel Émile. Paul Durel, (33 ans) et Étienne Auguste Esnout (32 ans).

111 Marins disparus et décédés. Juillet 1905. Brig Roland. Auguste Desjardins (26 ans).

112 Marins disparus et décédés. 28 avril 1884. Goelette Paquebot No 2. Saint-Servant.

113 Marins disparus et décédés. Juillet 1905. Para. Pierre Gervais (43 ans)

114 Marins disparus et décédés. 17 juin 1928. Trois-mâts Viana. Marcel-Augustin Donne (24 ans).

115 Marins disparus et décédés. 25 mai 1882. Goélette J.B.C. VJM Bequet.

116 Marins disparus et décédés. Pirogue № 309. Pierre Beaudouard. Non daté.

117 Marins disparus et décédés. 23 mars 1893. Trois-mâts Valleda. Pierre Cosentin Delepine (34 ans).

118 Marins disparus et décédés. 23 janvier 1928. Chalutier Caspian. Gustave Carlson. C'est une fin tout aussi tragique pour François Coupard, 61 ans, de Granville, assassiné à son domicile de l'Île-auxChiens en décembre 1888. Il était dans l'archipel depuis le 16 octobre. Marins disparus et décédés. 30 décembre 1888. François Coupard. L'hivernant Jean-Louis Daguerre, lui, de Saint-Jean-de-Luz, disparait en wary en 1877 alors qu' « étant en promenade ». Marins disparus et décédés 1877 (n.d.) Jean-Louis Daguerre.

119 Marins disparus et décédés . 10 janvier 1844. Pierre François Coepel.

120 Marins disparus et décédés . 28 novembre 1897. James Cusick.

121 Marins disparus et décédés . 29 septembre 1866. Jean-Baptiste Ernouf (35 ans).

122 Marins disparus et décédés 7 mai 1881. Transport Indre. JeanBaptiste Autier.

123 Voir les nombreux travaux de Marcus Rediker pour le $19^{\mathrm{e}}$ siècle. Les hors-la-loi de l'Atlantique : pirates, mutins et fibustiers, Paris, Éditions 
du Seuil, 2014.

124 Marins disparus et décédés . Hôpital de Reykjavik. Chalutier Notre Dame de Lourdes. Auguste-Marie Cressant, 33 ans, de Dinan.

125 Marins disparus et décédés . 9 mars 1883. Trois-mâts Gustave Adolphe. Auguste Célestin Bertrand.

126 Marins disparus et décédés . 2 avril 1900. Vapeur postal Patria. Jean-Baptiste Bouder, de Paimpol.

127 Marins disparus et décédés . 8 octobre 1894 et 6 juillet 1896 . Goélette L.H.B. Sévère François Auguste David (26 ans).

128 Marins disparus et décédés . 29 août 1894. Goélette Marie Louise. Henri François Desbois (19 ans).

129 Marins disparus et décédés . 24 juillet 1888. Brig-goélette Albertina. Granville. L.J.D. Delalande (44 ans). Voir aussi 12 septembre 1897. Goélette Saint-Pierrais. L.J.D. Delalande.

130 Marins disparus et décédés . Août 1881. Goélette Ernestine. Z.F. Guillet (33 ans). Voir aussi 1 mai 1884. Goélette Georges. Z.F. Guillet.

131 Marins disparus et décédés. 7 mai 1890. Goélette Joséphine. F.-M. Auffray. Voir aussi août 1908. Goélette Anita. F.M. Auffray. 30 juin 1912. Navire Casimir Périer. F.M. Auffray.

132 Marins disparus et décédés. Août 1923. Trois-mâts Michel Boisselier. E.J. Dubois. Voir aussi 8 juin 1929. Trois-mâts Santa Maria. E.J. Dubois.

133 Commandant L. Beaugé, chargé de mission, Grande Pêche. Rapport de mission sur le banc de Terre-Neuve (campagne de 1927), $3^{e}$ partie, Paris, L'Office des pêches maritimes de France, p. 39-42. Cette étude révèle que de 1915 à 1925, 32 navires s'échouent ou disparaissent « dans les eaux de la colonie ». Des 26 « sinistres complets ", trois sont dus à des voies d'eau dans le voisinage de la côte, trois à des manœuvres manquées, deux à une « chasse au mouillage » et 13 à la brume. 